\title{
AMBA: Adaptive Monarch Butterfly Algorithm based Information of Transfer Scheduling in Cloud for Big Information Application
}

\author{
D. Sugumaran ${ }^{1}$ \\ Associate Professor, Department of Information \\ Technology, Vel Tech Rangarajan Dr. Sagunthala R\&D \\ Institute of Science and Technology \\ Chennai,Tamilnadu,India
}

\author{
C. R. Bharathi ${ }^{2}$ \\ Associate Professor, Department of Electronics and \\ Communications, Vel Tech Rangarajan Dr. Sagunthala \\ R\&D Institute of Science and Technology \\ Chennai,Tamilnadu,India
}

\begin{abstract}
In present days cloud computing is most famous innovation and has a great deal of research potential in different zones like allocation of resource, scheduling of data transfer, security as well as privacy and so on. Data transfer Scheduling is one of the significant issues for improving the proficiency of all cloud based administrations. In cloud computing, data transfer scheduling is utilized to allot the task to best reasonable asset for execution. There are various types of data transfer scheduling algorithms. A few issues like execution time, execution cost, high delay time, complexity, and high data transfer cost as well as various optimization problems have been measured in existing papers. To tackle all the above problems, in this paper, a new Adaptive approach are introduced which is a combination of Monarch Butterfly and Genetic (AMBA) Algorithm based data transfer scheduling is proposed. So here the concept is to develop an optimal algorithm for scheduling the data transfer in an efficient way which helps in reducing the data transfer time. The performance of proposed methodology analyzed in terms of evaluation metrics.
\end{abstract}

Keywords-Information transfer scheduling; AMBA; throughput maximization; migration operation

\section{INTRODUCTION}

Cloud computing platform could be defined as the usage of computing assets for example Software as well as Hardware, Which the clients get them in type of administration through a system (regularly web). It goes for spreading enormous scale segments and assets that are required away, learning, calculation and data for scientific inquire about after some time, cloud applications are slanting more to rely upon system in the regions of intuitiveness or information access and furthermore their requests for prerequisites are expanding step by step. Calculation is mentioned by some specific errands which are referred to as employments and dictated by calculation, organize limit and capacity. Cloud applications utilize numerous VMs in the preparing of those information volumes.

Along these lines, such applications deal with various employments where they are done by accessible assets so the best results, briefest reaction time, most brief time of finishing and utilization of assets can be gotten (1) (2). It is a web based processing that gives assets respect to a compensation for each utilization premise. Because of the upsides of high figuring force, low administrations cost, better execution, adaptability, openness just as accessibility it has turned into a utility. It is separated into application, stockpiling and availability sections. Each section fills for different needs and gives items to organizations and peoples in the world. Without establishment retrieve their personal or official documents at any PC, it enables purchasers and organizations to access applications using internet. Virtualization is a basic component of distributed computing. It is programming that isolates physical foundations to make different assets (3) (4). The primary preferred position of job scheduling computation is to accomplish a superior figuring and the best framework throughput. Planning oversees accessibility of CPU memory and great scheduling strategy gives most extreme usage of asset (5).

The ruler butterfly improvement (MBO) calculation has demonstrated to be a successful apparatus to tackle different sorts of optimization problems. In any case, in the fundamental MBO calculation, the search methodology effectively falls into local optima, causing premature convergence as well as poor presentation on numerous complex optimization issues. It can diminish an arranging task in addition to improve the computational productivity (6). Overcoming MBO limitations, a selfish algorithm was introduced in genetic migration and genetic modification, as well as a method linked to the work of genetic scientists, maintaining a balance between geographical diversity and integration. Local (7). Getting a new position depends on how long you pay for a solar compass or an attractive compass, however most depend on how long the compass takes. New generation immigrants are produced by high magnetic forces and follow the previous generation where they go and the best features of any generation will continue to be passed on to the next generation (8) (9). FF can simply achieve the global optimum and it has solves the issues quick and it effectively flexible to the applications (10).

In this work, we are utilizing another new scheduling concept to transfer data in efficient manner dependent on Adaptive Algorithm. Our proposed adaptive algorithm powerfully solves the data transfer scheduling struggles. The aim is to build up a scheduling algorithm to transfer data utilizing a adaptive approach Consolidating monarch butterfly 
as well as genetic algorithm (AMBA) in cloud computing platform it find out best scheduled path to reduce data transfer time. The MB is a recently used algorithm and its basic nature is to solve global operational problems very quickly, and this algorithm is perfectly suited to similar processing and is well suited to the trade-off between durability and variability. Butterflies are in better health than their parents. This improves performance and speeds up the efficiency of data transfer planning. Lastly, data editing performance is analyzed based on different test metrics.

The subsequent content is in the order of; the proposed approach based literature survey is given in Section 2 and information transfer scheduling model is given in Section 3. The proposed optimal data transfer scheduling is explained in Section 4 and result and discussion is given in Section 5 and the conclusion part is in Section 6.

\section{LITERATURE SURVEY}

Alex X. Liu (11) uses multiple immensity data transfers scheduling. In this bulk data migration among data centres was frequently a significant stage in deploying new services, improving dependability underneath failures, or executing an assortment of cost reduction methodologies for cloud organizations. These immensity amounts of data transferring consume enormous transfer speed as well as, further cause extreme system blockage. To beat these above downsides, here, they explored the Multiple Bulk Data Transfers Scheduling (MBDTS) issue to diminish the system blockage or network congestion. Transiently, they applied the storeand-forward exchange mode to lessen the pinnacle traffic load on the connection.

Roman Barták (12) Roman Barták (12) utilizes MAPF it manages the issue of finding a collision free path for a lot of operators. A Scheduling Based Approach to Multi Agent Path Finding with Weighted as well as Capacitated Arcs The real inspiration for the scheduling model of MAPF was its ability to normally incorporate different limitations. They considered especially the issues, where the limit of arc scan was more prominent than one that is more specialist output utilizes a similar curve in the meantime, and the lengths of circular segments was greater than one that is moving between various sets of nodes takes various occasions' times. These augmentations make the model nearer to reality than the original MAPF formulation.TevfikKosar (13) utilizes Dataaware scheduling in grid computing. This was Efficient as well as dependable access to enormous scale information sources along with documenting goals brings new challenges in widely scattered computing environment. The deficiency of the conventional frameworks as well as existing CPU-situated cluster schedulers in tending to these difficulties has yielded another emerging era: data aware schedulers. Here, they examine the limitations of the conventional CPU in handling the difficulty of demanding data management in wide ranging of distributed applications. Saurabh Kumar Garg (14) HPC clients need the capacity to increase quick and adaptable accessing to high performance computing abilities. Cloud computing guarantees to convey such kind of infrastructures utilizing data centres, by these HPC clients can use applications as well as access the information through Cloud from anywhere in the world. In any case, due to increase in the demand which drastically expands the vitality utilization of data centres, which has turned into a basic issue. High vitality utilization not just means high vitality cost which was decreased the overall revenue of Cloud suppliers, yet in addition high carbon emanations which was not suitable for environment. To tackle this problem, they introduced nearoptimal scheduling strategies that exploit heterogeneity over various server farms for a Cloud provider.Yuan Zhang (15) uses a planning strategy that consists of two sections. With the allocation of a computer component, which is part of a longterm job measurement, planning can divide the work process into task categories by mandatory data transfer, because each Task Team is assigned a computer process. The unit that completed the task team at the most convenient time. Instead of simulation and wireless communication, they simply use the robin circular rule. The process of measuring the phase release was discussed, according to the performance phase analysis. In this case, the outcome indicates that the proposed resource planning strategy may be delayed.

\section{INFORMATION TRANSFER SCHEDULING}

Information transfer scheduling is used to transfer the information from source to destination leads to minimum time and maximum throughput. In the cloud, information is stored in different nodes, not all information is stored in local servers, and some of the nodes may have to fetch information from distance servers. In this case, time may be increased. So, to avoid time consuming, replicas are generated. In this manner, needed information can be fetched from one of the replica servers. In the information retrieval process, initially, the node is chosen for information recovery, an information transfer path is specified from the requesting node to the information transfer node. A lot of paths are available for one transmission. Among them, we will choose the one shortest path which wills lead to minimize the time and maximize the throughput. If we select the multi-path means, the system will suffer from high jitter. A naive strategy is to choose hubs and paths arbitrarily, however, it might outcome in overwhelming congestions on certain connections, prompting long information recovery time, since it not consider link bandwidths and the overlaps of chosen paths and hubs. Information transfer based on the randomly chosen path is given in Fig. 1. To overcome the problem the shortest part is optimally selected with the help of hybridization approach. The optimal information transfer scheme is given in Fig. 1.

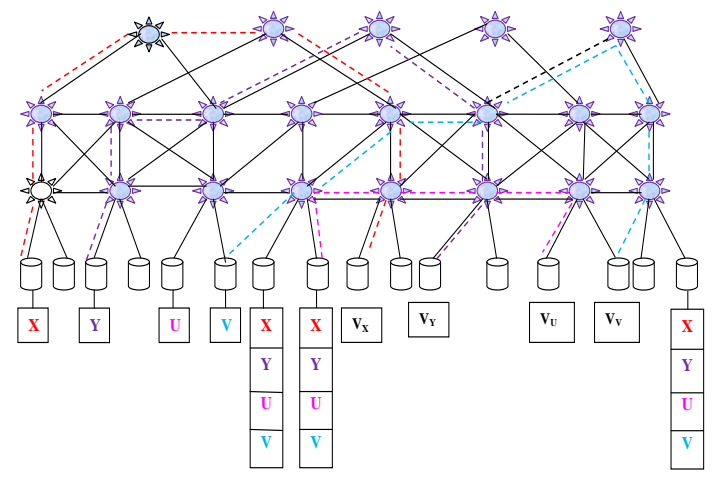

Fig. 1. Information Transfer based Randomly Chosen Path. 


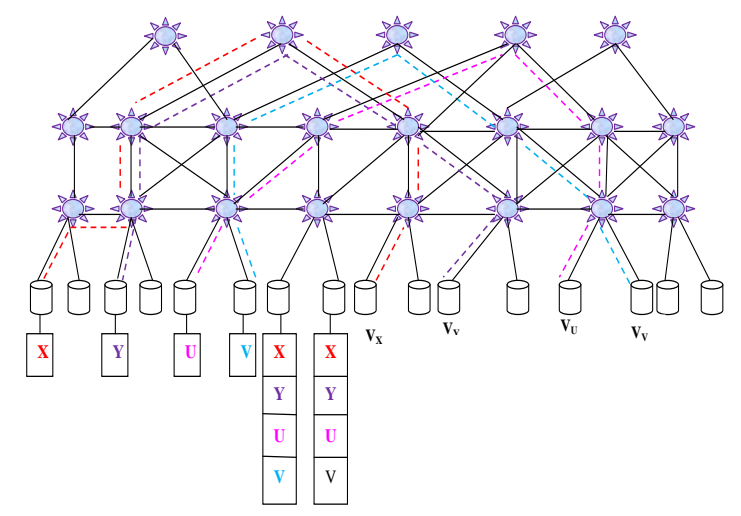

Fig. 2. Optimal Information Transfer Scheme.

In Fig. 2, $\mathrm{X}, \mathrm{Y}, \mathrm{U}$ and $\mathrm{V}$ are the information and $V_{x}, V_{y}, V_{u}$ and $V_{v}$ are retrieving hubs. Hub $V_{x}$ retrieving information $\mathrm{x}$ which is specified as red dash line, $V_{y}$ is retrieving information $\mathrm{y}$ which is specified as violet dash lines, $V_{u}$ is retrieving a information $\mathrm{u}$ which is represented as pink dashed lines and $V_{v}$ retrieving a information $\mathrm{v}$ which is specified as blue dashed lines. In Fig. 2, both data transfers share common traffic links at high volume and can lead to higher transmission times for the lower 2 numbers to pass in batches, resulting in shorter data retrieval. It is still distributed among computing nodes, or all data is accessible, so few sites may need to access data from remote locations. In this case, the requested information can be obtained at one of its locations. At that time, when a node is preferred for data acquisition, the request method for that requesting node requires specification of the data transfer. Let as consider an example of individual system, in a polygon as shown in Fig. 1, the four information elements (X, Y, U and $\mathrm{V}$ ) are stored in 4 duplicates, and each link has a bandwidth of data per second. Note that it takes at least a second to move data between two centers.

Take simultaneously, the node will retrieve data $V_{x}$, retrieve data $\mathbf{x}, V_{y}$ retrieve data $\mathbf{y}, V_{u}$, and retrieve data $V_{v}$, both active and random can have a information retrieval time of 4 seconds; although a good solution takes a second. The idle method works poorly, because all data transfers go through the normal connection, causing the bottle to be shown in Fig. 1. Less time was taken by correct solution because node selection and all data transfers with sets Links are not shown in Fig. 2 Network (DCN) shown in Fig. 3.

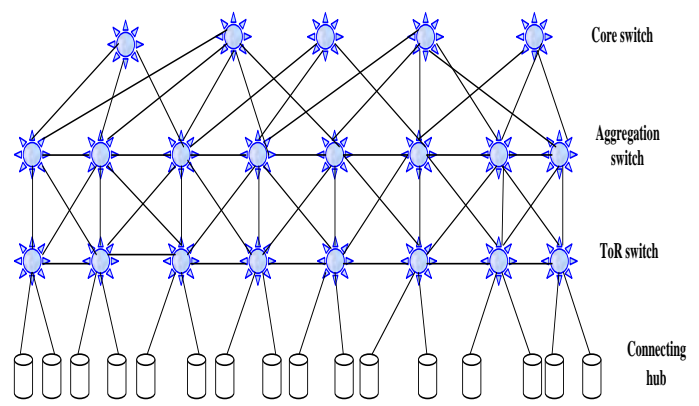

Fig. 3. Information Centre Network Topology.

\section{PROPOSED INFORMATION TRANSFER SCHEDULING SYSTEM}

In this work, we have intended to enlarge an information transfer scheduling scheme based on Adaptive Algorithm. This adaptive approach finds the best path based on the least information transfer time-the maximum throughput. The aim is to develop an optimal information transfer scheduling using an Adaptive approach combining monarch butterfly (MB) and genetic operators (AMBA) in the cloud computing environment. The proposed methodology structure is given in Fig. 4.

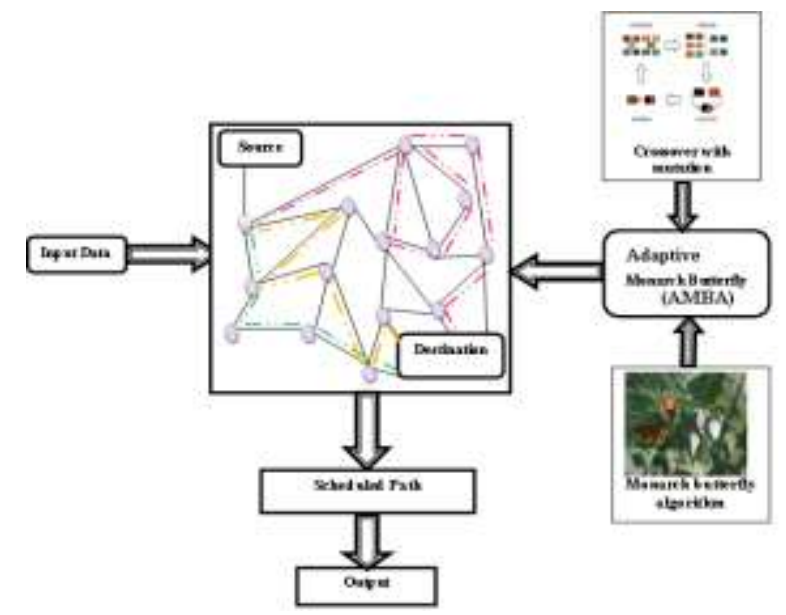

Fig. 4. Proposed Methodology Structure.

The proposed MBA is a recently developed optimization algorithm which is mainly used for solving global optimization problem (1). Basically, the MBA has two operators, namely migration and adjusting. But in basic MBA, we have some difficulties in the search process conducted by the butterfly adjusting operator. Early stages of algorithm's execution, the search process that is exceedingly directed towards the current best solution in the population, in some of the algorithm's run generate poor results. To tackle with this deficiency, MBA is adaptive with genetic operators. The step by step process of AMBA based optimal path selection process is explained below:

\section{Step 1: Initialization phase}

Solution initialization is a chief process for the entire optimization problem. Here, the initial solution is initialized at random. Initially, we initialize the solution parameters. For data retrieval process, $\mathrm{N}$ number of the path is available. To increase the speed and minimize the transformation time and cost, we optimally choose the path. In this paper, the path is considered as a monarch butterfly (solution) and the population is considered as a butterfly. Initially, monarch butterflies are randomly initialized.

Consider information $\mathrm{x}$ and $\mathrm{y}$ is transferred to hub $\mathrm{u}$ and $\mathrm{v}$. Here, for security purpose, we have generated three replicas for both information $\mathrm{x}$ and $\mathrm{y}$. The system generates multiple. The initial solution format is given below:

$S_{i}=\left(P_{1}, P_{2}, \ldots ., P_{N}\right)$ 
After that, we divide the population into two groups namely subpopulation $1\left(\mathrm{SP}_{1}\right)$ and subpopulation $2\left(\mathrm{SP}_{2}\right)$. NP represented the total number of monarch butterflies. The number of MB's present in $\mathrm{SP}_{1}$ is calculated using equation 2.

$N P 1=\operatorname{ceil}(p \times N P)$

The number of MB's present in $\mathrm{SP}_{2}$ is calculated using equation 3 .

$N P 2=N P-N P 1$

Where;

$\mathrm{p}->$ migrating speed of monarch butterflies with $p=5 / 12$ in $\mathrm{MB}$,

Ceil(x) rounds $\mathrm{x}$ to the nearby integer larger than or equivalent to $\mathrm{x}$,

Step 2: Fitness calculation

After the solution initialization, the fitness of each butterfly is calculated. The fitness function is based on cost and time. If the butterfly attains the minimum cost and time means that the butterfly is considered as the best fitness.

\section{Fitness $=\min (\cos t$, time $)$}

Step 3: Migration operation

After the fitness calculation, each butterfly migrates their position. The migration function can be written as follows:

$S_{i}^{q+1}=\left\{\begin{array}{l}S_{r 1}^{q}, r \leq p \\ S_{r 2}^{q}, r>p,\end{array}\right.$

$S_{i}^{q+1}$ is a k-th part of $S_{i}$ in generation $q+1$; similarly, $\boldsymbol{S}_{r \mathbf{1}}^{\boldsymbol{q}}$ denotes the k-th fraction of $\boldsymbol{S}_{\boldsymbol{r 1}}$ in generation $\mathbf{q}$, and $S_{r 2, k}^{q}$ q is the k-th fraction of $S_{r 2}$ in generation q; the current generation number is q, and the monarch butterflies $\mathrm{r} 1$ and $\mathrm{r} 2$ are randomly selected from subpopulation 1 and subpopulation 2, so here, $\mathrm{r}$ is calculated by $\boldsymbol{r}=\boldsymbol{r} \times \boldsymbol{p e r i}$, where peri is the time of migration, which is 1.2 in $\mathrm{MB}$ and the rand is a random number in $(0,1)$.

\section{Step 4: Adjustment operation}

After migration operation, adjustment operation is done in $\mathrm{SP}_{2}$. The subsequent formula is described as,

$S_{i}^{q+1}=\left\{\begin{array}{c}S_{\text {best }}^{q} \text { rand } \leq p \\ S_{r 3,}^{q} \text { rand }>p, \text { rand } \leq B A R \\ S_{i}^{q+1}+\alpha \times\left(d s_{k}-0.5\right), \text { rand }>p \text {, rand }>B A R,\end{array}\right.$
In any case, $S_{i}^{q+1}$ is part of the k-th of $S_{j}$ in generation q + 1; Similarly, $S_{\text {best }}^{q}$ is the k-th part of $S_{\text {best }}$ in generation q, which is the best place for monarch butterflies in world 1 and land 2, $S_{r 3, k}^{q}$ part $S_{r 3}$ in generation q, king butterfly r3 selected at random in the case of less than 2, and BAR is the conversion or correction rate, if $\mathrm{BAR}$ is less than the random number $\mathrm{r}$ and the $\mathrm{k}$-th fraction of $x_{j}$ at $q+1$, when $\sigma$ is measured, and $\sigma=S_{\max } / q^{2}$, where $S_{\max }$ is the highest travel step.

\section{Step 5: Crossover operator}

After the migration process, to improve the MBA, an additional operator is integrated with the MBA. Crossover is the process by which genes are selected from the chromosomes of parents and new offspring. Crossover can be done with binary code codes, coding code, pricing code and encoding Fig. 5.

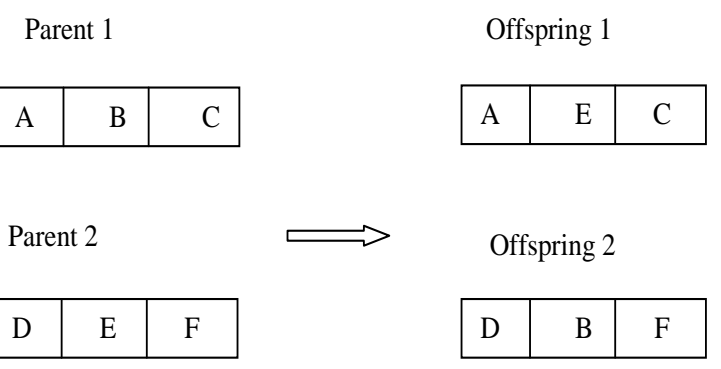

Fig. 5. Crossover Process.

\section{Step 6: Mutation operator}

After skipping a task, the solution is updated with the help of modifications. Genetic modification function, can search for new locations in contrast to the crossing. The crossover is called the exploit operator and the conversion is a form of proof Fig. 6.

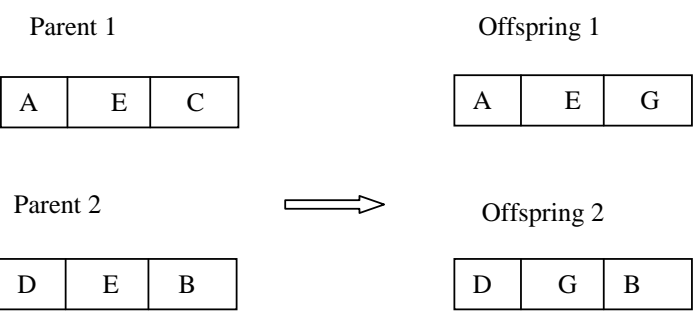

Fig. 6. Mutation Operator.

Step 7: Termination criteria

The algorithm stops its use only when selecting the highest frequency selection and the solution contains the best body weight and is given as the best option. The AMBA based optimal path selection algorithm is given below. 


\section{Algorithm}

Input: parameter of MBA, Cross over rate, mutation rate, number of hub, sender, destination, number of replicas

Output: Optimal path

Start

1. Randomly initialize the number of paths available in data transfer

2. Divide the whole population into two SP1 and SP2

3. Calculate the fitness for each path (monarch butterfly)

4. While $\mathrm{k}<\mathrm{MaxGen}$ do

5. Sort all individual in the population based on the fitness value

6. For $i=1$ to SP1 (all butterflies in the SP1) do

7. Apply butterfly migration operation to get a new butterfly

8. End for

9. For $\mathrm{j}=1$ to SP2 (all butterfly present in the SP2) do

10. If $\mathrm{t}<$ Maximum generation 0.5 then

11. Generate new butterfly in SP2 by using cross over and mutation operator

12. Else

13. Generate new butterfly in SP2BY using butterfly adjustment operator

14. End if

15. End for

16. Merge SP1 and SP2

17. Increase the iteration counter $\mathrm{k}$ by one

18. End while

19. Return bet butterfly in the whole population.

20. Output

Optimal path

\section{Simulation AND RESUlts}

In this section, the proposed information transfer scheduling scheme based on adaptive Algorithm. Our proposed adaptive algorithm efficiently solves information transfer scheduling problems. In this paper, we used an adaptive approach which integrating the monarch butterfly algorithm and Genetic algorithm (AMBA) in the cloud computing environment.

\section{A. Experimental Results}

While testing the AMBA algorithm performance, it may be difficult to verify the functionality of all the same algorithms. The main use of this to solve the problems of speeding up the earth again, this algorithm is perfectly fit for similar processing and can trade between durability and variability. In the proposed work, finally, the effectiveness of the data transfer planning process is analyzed according to different test criteria. Comparing to the existing optimization techniques our proposed AMBA achieves the better results. The following Fig. 7, 8, 9 and 10 shows the waiting time, turnaround time, response time and fitness of the proposed approaches.

Analyzing Fig. 7, 8, 9, and 10 show the comparative analysis of proposed against existing based on waiting time, turnaround time, response time and fitness. Analyzing Fig. 7 our proposed AMBA algorithm achieves the minimum waiting time of $865,1245,1625$, and 2248 for other existing optimization and without optimization algorithms because Initially it calculates the jobs entirety completion time on every computing node, as well as file access time or duplication time among stored files along with every Computing node file size, bandwidth and the waiting time for every job earlier than it process and processing time. The above Fig. 7 clearly specifies our proposed approach waiting time is minimum for comparing all the other existing systems. Fig. 8 turnaround times is the total time taken among the whole process which is measured by the time interval from starting time and completion time of the process. Analyzing Fig. 8 our proposed AMBA algorithm achieves the minimum time of $1985 \mathrm{~ms}, 2635 \mathrm{~ms}$, 3845ms and $4958 \mathrm{~ms}$ for other existing MBO, GA and without optimization techniques.

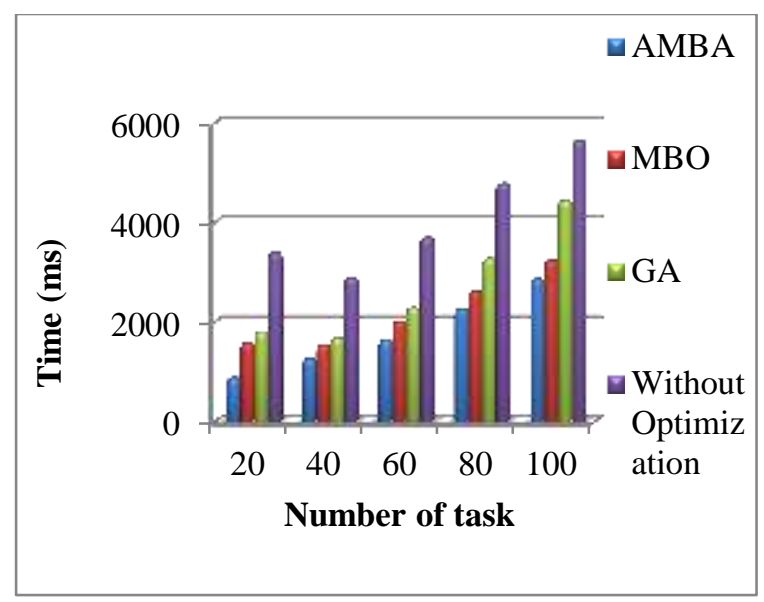

Fig. 7. Waiting Time Analysis.

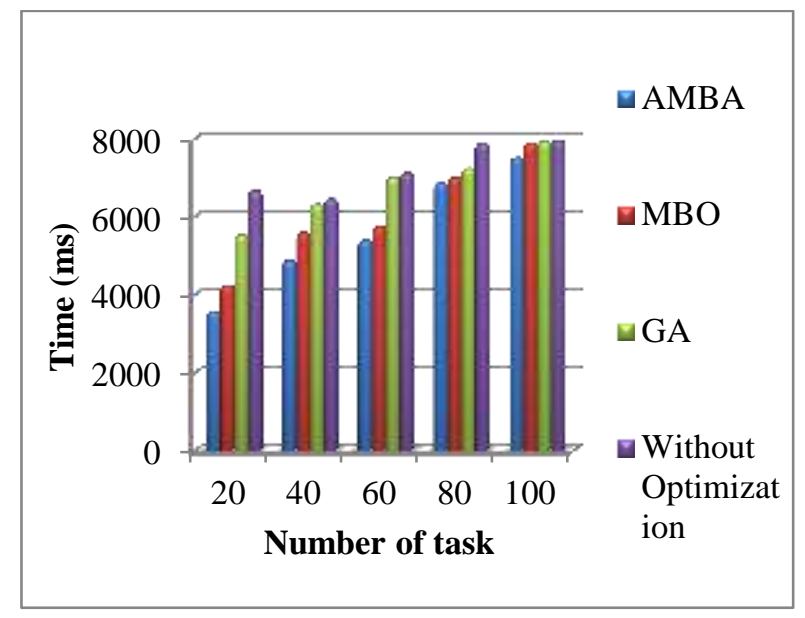

Fig. 8. Turnaround Time Analysis. 


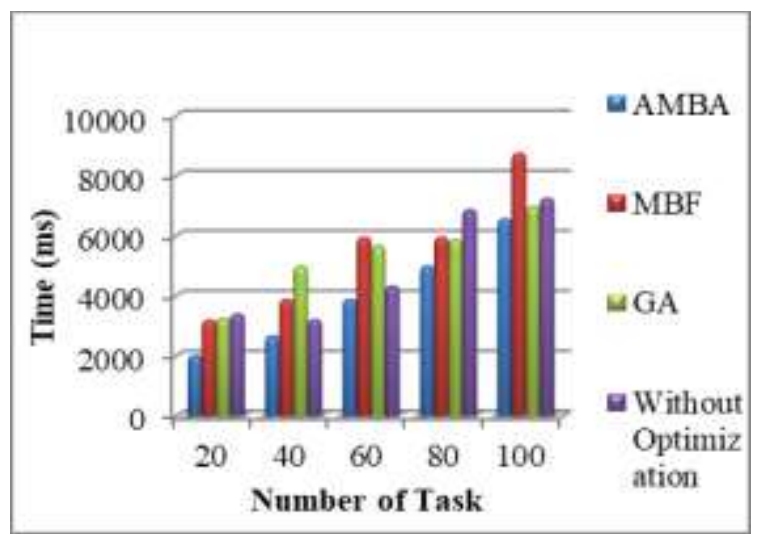

Fig. 9. Response Time Analysis.

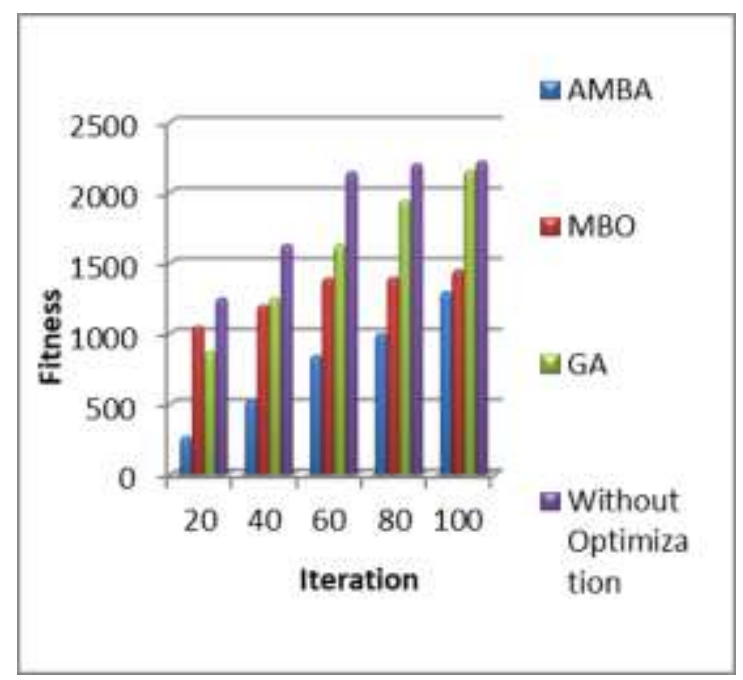

Fig. 10. Fitness versus Iteration.

Fig. 9 shows the comparative analysis based on AMBA it minimizes the response time. Our proposed AMBA achieves the minimum response time of $3545 \mathrm{~ms}, 4854 \mathrm{~ms}, 5365 \mathrm{~ms}$, and $6847 \mathrm{~ms}$ for other existing optimization and without optimization algorithms because the time taken to process a user request from the source point it is made up till the destination point it is received. Comparing to all the other existing techniques, the above Fig. 9 clearly specifies our proposed approach response time is minimum for comparing all the other existing systems.

Fig. 10 our proposed AMBA minimize the objective function value of $1985,2635,3845$, and 4958 for other existing optimization and without optimization algorithms.

Comparing to all the other existing techniques, AMBA it minimizes the fitness of objective function values. Experimental studies have shown that our proposed AMBA is better than other available solutions for full durability and delay because genetic operators are used in MB in migration operation, and this built-in strategy resides only with people with better potential than their parents. The performance will be improved and it speeds up the efficient data transfer process. In the proposed project, a genetic operator is used in MB to do migration work, and this built-in strategy can only accommodate monarch butterfly people who are more highly trained than their parents. Computer results indicate that the proposed process exceeds existing methods. Individual power is used as the purpose function of the solution for the same system solution. At the same time the solution reduces the amount of meaningful work, the best that can be achieved. From the test results, we are well aware that our proposed method achieves better results compared to existing methods.

\section{CONCLUSION}

In this paper, a new adaptive Monarch Butterfly and Genetic operator (AMBA) Algorithm could be proposed, make use of this algorithm the information transfer scheduling problems could be solved efficiently. The aim of research is to improve the best data transfer planning using AMBA on a cloud platform where you will find the most optimized method which leads to the shortest time of data transfer; in other words, the highest number. In this case, MB is one of the proposed algorithms that will be used later. The main application of this to solve the problems of re-accelerating the earth, this algorithm is perfectly suitable for the same processing and is able to trade between durability and variability. The genetic operator can easily access global operations and solve problems quickly and easily adapt to applications. In the proposed work, the GA applies methyl bromide in migration operations, and this included strategy can only accept monarchs that are healthier than their parents. The performance will be improved and it speeds up the efficiency of data transfer planning. Finally, our proposed approach outperformed other existing MBO, GA and without optimization approaches based on waiting time, TAT, response time as well as Fitness.

\section{REFERENCES}

[1] EsmaYildrim,EnginArslan,Jangyoung Kim, and TevfikKosar,In IEEE," Application level optimization of big data transfer" IEEE, March (2015).

[2] Aakanksha Sharma, Sanjay Tyagi, "Task Scheduling in Cloud Computing" International Journal of Scientific \& Engineering Research, Volume 7, Issue 12, December-2016.

[3] D. A. Agarwal and S. Jain, "Efficient Optimal Algorithm of Task Scheduling in Cloud Computing Environment," International Journal of Computer Trends and technology (IJCTT), 2014.

[4] M. Kalra and S. Singh, "A review of Metaheuristic Scheduling Techniques in cloud computing," Egyptian Informatics Journal, Elsevier, 2015.

[5] E. Kumari and M., "A Review on Task Scheduling Algorithms in Cloud Computing," International Journal of Science, Environment and Technology, 2015.

[6] LinSun, JiuchengXu, "Improved Monarch Butterfly Optimization Algorithm Based on Opposition-Based Learning and Random Local Perturbation", 10 February 2019.

[7] Shifeng Chen, Rong Chen, "A Monarch Butterfly Optimization for the Dynamic Vehicle Routing Problem", Department of Information Science and Technology, Dalian Maritime University, 12 September 2017.

[8] Kaushik Kumar Bhattacharjee, S.P. Sarmah, "Monarch Migration Algorithm for Optimization Problems", Department of Industrial \& Systems Engineering, Proceedings of the 2015 IEEE IEEM.

[9] P. Guerra, C. Merlin, R. Gegear, and S. Reppert, "Discordant timing between antennae disrupts sun compass orientation in migratory monarch butterflies," Nature Communication, vol. 3, p. 958, 2012.

[10] Umar SHEHU, Safdar, Gregory, "Fruit Fly Optimization Algorithm for Network-Aware Web Service Composition in the Cloud", (IJACSA) International Journal of Advanced Computer Science and Applications, Vol. 7, No. 2, 2016. 
[11] Sen Su, Zhongbao Zhang, "Multiple bulk data transfers scheduling among data centres",Department of Computer Science and Engineering, Michigan State University, 18 February 2014.

[12] Roman Barták, Marec VLK, "A Scheduling-Based Approach to MultiAgent Path Finding with Weighted and Capacitated Arcs", Scheduling and Planning, AAMAS July 10-15 2018.

[13] TevfikKosar, Mehmet Balman, "A new paradigm: Data-aware scheduling in grid computing", Department of Computer Science, Elsevier 2009.
[14] Saurabh Kumar Garg, Chee Shin Yeo, "Environment-conscious scheduling of HPC applications on distributed Cloud-oriented data centers", Cloud Computing and Distributed Systems Laboratory, Department of Computer Science and Software Engineering, Elsevier 2010.

[15] Yuan Zhang, Nanjing, "Resource Scheduling and Delay Analysis for Workflow in Wireless Small Cloud", IEEE Transaction on Mobile Computing, volume: 17, Issue: 3, March 1, 2018. 